\title{
Rapid Assessment of Homogeneity and Stability of Amorphous Solid Dispersions by Atomic Force Microscopy-From Bench to Batch
}

\author{
Matthias E. Lauer • Monira Siam • Joseph Tardio • Susanne Page • Johannes H. Kindt • Olaf Grassmann
}

Received: 3 October 2012 / Accepted: 1 April 2013 / Published online: 15 May 2013

(C) The Author(s) 2013. This article is published with open access at Springerlink.com

\begin{abstract}
Purpose To verify the robustness and fundamental value of Atomic Force Microscopy (AFM) and AFM-based assays to rapidly examine the molecular homogeneity and physical stability of amorphous solid dispersions on Hot-Melt-Extrudates.

Methods Amorphous solid dispersions were prepared with a Hot-Melt Extruder (HME) and profiled by Raman Microscopy and AFM following a sequential analytical routine (Multi-ScaleImaging-of-Miscibiliy (MIMix)). Extrudates were analyzed before and after incubation at elevated temperature and humidity. The data were compared with published results as collected on miniaturized melt models. The value of molecular phase separation rates for long term stability prediction was assessed.

Results Data recorded on the extrudates are consistent with those published, and they can be compared side by side. Such direct data comparisons allow the identification of possible sources of extrudate heterogeneities. The surface roughness analysis of fracture-exposed interfaces is a novel quantitative way to trace on the nanometer scale the efficiencies of differently conducted HME-processes. Molecular phase separation rates are shown to be relevant for long term stability predictions.

Conclusions The AFM-based assessment of API:excipient combinations is a robust method to rapidly identify miscible and stable solid dispersions in a routine manner. It provides a novel analytical tool for the optimization of HME processes.
\end{abstract}

KEY WORDS amorphous solid dispersion · atomic force microscopy · hot melt extrusion · process optimization . stability prediction

$\begin{array}{ll}\text { ABBREVIATIONS } \\ \text { AFM } & \text { Atomic Force Microscopy } \\ \text { API } & \text { Active Pharmaceutical Ingredient } \\ \text { CETP(Inh) } & \text { CETP Inhibitor } \\ \text { DSC } & \text { Differential Scanning Calorimetry } \\ \text { Eudragit LI00 } & \text { Polymethacrylate } \\ \text { HME } & \text { Hot-Melt-Extrusion } \\ \text { HPMCAS MF } & \text { Hydroxypropyl methylcellulose acetate } \\ & \text { succinate } \\ \text { MIMix } & \text { Multi Scale Imaging of Miscibility } \\ \text { NKI(Ant) } & \text { NKI Antagonist } \\ \text { PHR } & \text { Phase Homogeneity Ratio } \\ \text { PVP K30 } & \text { Polyvinylpyrrolidone K30 } \\ \text { PVP VA 64 } & \text { Copolymer of I-vinyl-2-pyrrolidone and } \\ & \text { vinyl acetate } \\ \text { PVPPF I7 } & \text { Polyvinylpyrrolidone PFI7 } \\ \text { QCMM } & \text { Quench-Cooled-Melt-Mixture } \\ \text { RH } & \text { Relative humidity } \\ \text { RI } & \text { Raman Imaging } \\ \text { SEM } & \text { Scanning Electron Microscopy }\end{array}$

Electronic supplementary material The online version of this article (doi: I 0. I007/s I 1095-0 I3- |045-0) contains supplementary material, which is available to authorized users.

M. E. Lauer $(\bowtie) \cdot M$. Siam • O. Grassmann

F. Hoffmann-La Roche Ltd., pRED

Small Molecule Research, Discovery Technologies

4070 Basel, Switzerland

e-mail: Matthias.Lauer@roche.com

J. Tardio $\cdot$ S. Page

F. Hoffmann-La Roche Ltd., pRED

Small Molecule Research, Formulation Research

4070 Basel, Switzerland
J. H. Kindt

Bruker Nano GmbH Oestl. Rheinbrueckenstr. 49

$76 \mid 87$ Karlsruhe, Germany 
$\mathrm{T}$

TEM

Temperature

Transmission Electron Microscopy

\section{INTRODUCTION}

Amorphous solid dispersions can increase the oral bioavailability of poorly water soluble drugs. The drug molecules are intended to be conserved amorphously in a matrix, typically of polymeric excipients, ideally in the form of homogenously dispersed, dissolved molecules. Single dispersed drug molecules do not have the physico-chemical properties widely associated with their bulk ensembles, crystals or other bulk volume phases. Many physical material properties are changed in an amorphous state, for example, the dissolution behavior, thermal and mechanical properties, spectroscopic properties and even the specific density of the material (1).

A well-known disadvantage of amorphous solid dispersions is the lack of thermodynamic stability, as small and poorly soluble drug molecules often have a high potential to crystallize out. One topic of fundamental research is to identify and to better understand amorphous drug-polymer systems which offer an appropriate and uniform dissolution performance invivo, and which also can be prevented from phase separation in the solid state on the long term. It is convenient to screen for such systems using miniaturized formulation models accessible by quench-cooling of drug-excipient melt mixtures $(2,3)$, or by various casting methods (4-7) .

Physical stability studies are usually time-consuming, as phase separation in the solid state is a comparatively slow physical process, its velocity depending on many factors, including the initial dispersion structure, thermal diffusion coefficients, the drug-polymer interaction and the corresponding phase mobilities, and the crystal nucleation energy of the drug. A critical amount of sufficiently sized drug and excipient phases needs to have formed to change the overall physical properties of a material significantly.

Many established analytical methods to routinely characterize amorphous solid dispersions examine properties which are relevant only to bulk packed molecular ensembles, rather than of dissolved molecules. X-ray powder diffraction (XRPD), for instance, typically verifies the nature and amount of diffracting crystals within already critical mixtures (8). Differential Scanning Calorimetry (DSC) typically inspects for the presence of heterogeneous bulk phase properties at a given temperature and time, with the initialorigin system constitution often unknown (9). Classical optical methods such as polarization microscopy and Raman Imaging reveal heterogeneity information of single regions with a resolution in the range of the wavelength of light $(10,11)$. None of these methods delivers information about the detailed spatial molecular structure of these systems, the phase homogeneity and properties on the nanometer scale.
None of these methods is intended to differentiate between the surface and the volume properties of a material. The highly complex nature of solid dispersions is typically characterized by the absence of physical properties potentially observable by complementary analytical methods (12). It is a widely appreciated analytical challenge to directly differentiate between similarly composed but differently prepared and performing amorphous solid dispersion systems using only the "bulk-averaging"analytical techniques (13).

High-Resolution Imaging Technologies provide information on the level of single particles rather than displaying the properties of larger material sections and bulk packed particle ensembles. Scanning Electron Microscopy (SEM) is preferably applied to image particles of complex morphologies with an inherently better resolution than classical optical microscopy (14). Atomic Force Microscopy (AFM) provides better spatial resolution on soft organic material surfaces, but is operatively restricted to rather smoothly shaped sample surfaces and small particles. Such cantilever-based methods have, however, their attractiveness as they allow the examination of molecular surface landscapes and surface properties in an ambient environment, directly and nondestructively. AFM data are metrological rather than photographic in nature and can, for instance, be easily quantified in respect to the molecular surface roughness (15).

Phase mapping, as it is routinely conducted with AFM under tapping-mode operations, is well established for the qualitative display of mechanical material heterogeneity contrasts (16). Tapping AFM can also be performed in an automated and high throughput manner. Other cantileverbased sensing modes (17), such as Peak Force Quantitative Mapping (18), nano-thermal AFM (19), or Nano-IR (20) can be applied when information beyond that of a qualitative binary phase heterogeneity is required.

AFM -based methods have been established to characterize amorphous systems (21-23). They were recently helpful to identify low quantities of phase-separated drugs in Hot-Melt-Extrudates (24), or to characterize the surfaces of particles obtained by spray drying (25). We have shown that AFMs can be applied in an assay manner, and for instance, two drug compounds could be recently profiled within a set of five polymeric excipients with respect to their molecular miscibility and physical stabilities in a parallelized and systematic workflow, MIMix (26).

MIMix utilizes fundamentally different imaging technologies (Raman Imaging and AFM) that are operated at different resolution regimes to visualize surface phase constitutions on the micrometer and on the nanometer length scales. MIMix comprises three basic and hierarchically organized analytical working steps. Firstly, MIMix examines fracture-exposed bulk interfaces for spatial-spectroscopic uniformity on the micrometer scale using confocal Raman Imaging. Secondly, these fracture-exposed molecular landscapes are probed with the AFM to determine the phase 
homogeneity status on the nanometer scale. The molecular morphology is determined on $3 \times 3 \mu \mathrm{m}^{2}$-sized surface sections and on different sample regions. The morphological deviation from the image mean plane is expressed in terms of a root mean square roughness, and the variability of different regions on the sample is expressed as the standard deviation. The mechanical property contrast is sensed by recording phase maps by AFM tapping-mode operation. These maps are additionally inspected for nanometer-sized features, which are indicative of separated phases. Only amorphous solid dispersions exhibiting a surface roughness and deviation similar to or smoother than that of the corresponding pure excipient blank, and which are also featureless in the phase images, are then considered to be of a molecular homogenous structure, and only those are here termed to fulfill the MIMix homogeneity criteria. Thirdly, the last MIMix step consist of stress tests (accelerated stability test) to finally determine and compare small-sized structural changes within the surface phase constitutions such as may introduced by applied environmental stress or storage time on a time scale of hours.

The assay and MIMix workflow was developed to examine the intrinsic melt miscibility and stability of a given drug within various excipients using freshly-fractured quenchcooled melt mixtures ("QCMM"). This novel AFM approach and the data gathered are encouraging, as they can provide a novel analytical way of better comparing and ranking differently composed dispersion systems in an objective fashion. This approach and its concepts, however, have at the moment not in general been proven to accelerate and facilitate formulation research on amorphous material.

It is currently unknown how structure-property relations determined from surfaces on the nanometer scale and using QCMMs might compare with those generated by established processes such as Hot-Melt-Extrusion. It is unclear to which extent phase separation rates examined on fracture-exposed bulk interfaces on the molecular scale are reproducible, how sensitive such surfaces structures are to environmental changes, and whether the results generally compare with those amorphous systems processed by different means. It is clear that these questions involve some methodological issues, as it is necessary to compare objectively amorphous surface structures of differently composed, structured or processed systems. Another fundamental requirement is to show that molecular phase separation rates determined within hours are predictive of long-term macroscopic material stabilities of dispersion systems processed by completely different means.

These unresolved issues were the motivation to process two previously profiled drugs, an NK1 Antagonist and a CETP inhibitor with a small-scale Hot-Melt Extruder, and to subject those to the previously proposed MIMix workflow (26). The extrudate data are compared side-by-side with those of the assay published previously.

\section{MATERIALS AND METHODS}

\section{Compound Selection}

The selection of Active Pharmaceutical Ingredients (APIs) and excipients was that of compounds screened previously (26). An NK1 Receptor Antagonist, which will be referenced to as NK1(Ant) (27), and a CETP Inhibitor (28), which will be referenced to as CETP(Inh) were selected there. NK1(Ant) has a melting point $\left(\mathrm{T}_{\mathrm{m}}\right)$ of $\mathrm{T}_{\mathrm{m}}=130^{\circ} \mathrm{C}$ : the melting point of $\operatorname{CETP}(\operatorname{Inh})$ is $\mathrm{T}_{\mathrm{m}}=$ $142^{\circ} \mathrm{C}$. NKl(Ant) has a higher tendency to crystallize and was known to be more difficult to stabilize than CETP(Inh). The glass transition temperature and crystallization tendencies are shown with Differential Scanning Calorimetry in Supplementary Material (29). The excipient selection focused on the polymer class and five different polymers were chosen: the copolymer of 1vinyl-2-pyrrolidone and vinyl acetate (PVP VA 64, BASF, Ludwigshafen, Germany); Polyvinylpyrrolidone K30 (PVP K30, BASF, Ludwigshafen, Germany); Polyvinylpyrrolidone PF17 (PVP PF17, BASF, Ludwigshafen, Germany); Hydroxypropyl methylcellulose acetate succinate type M (HPMCAS MF, Shin-Etsu, Tokyo, Japan) and Polymethacrylate (Eudragit L100, Evonik Industries AG, Essen, Germany). To enable the side by side comparison of the here prepared extrudates with the previously profiled QCMMs the same API:excipient ratio of $1: 1$ has been applied (26).

\section{HME}

The extrudates were prepared with a small-scale Hot Melt Extruder. A DSM Micro 5 Compounder (DSM Xplore, Geleen, The Netherlands) with co-rotating twin screw and $5 \mathrm{~mL}$ batch-volume with recirculation channel was used. The Extruder has 4 heating zones; upper and lower zone with independent front and rear adjustments. The drug-polymer mixtures were weighted in the selected proportion (1:1), gently mixed in a mortar (dry) and sieved through a $0.6 \mathrm{~mm}$ screen. $5 \mathrm{~g}$ of each physical blend mixture were loaded into the extruder at $120^{\circ} \mathrm{C}$ (all 4 zones) at a screw speed of $100 \mathrm{rpm}$ within $\sim 5$ min. Mixing was allowed during 5-10 min at $140^{\circ} \mathrm{C}$ (all 4 zones) and $100 \mathrm{rpm}$ by recirculation. The temperature was then adjusted to $120^{\circ} \mathrm{C}$ to increase the viscosity and the extrusion was conducted at a screw speed of $50 \mathrm{rpm}$. 


\section{Preparation of Extrudates for the RI and AFM Investigations}

For microscopic investigations fresh and smoothly fractured extrudate surfaces are required.Horizontally aligned fracture surfaces were generated after introducing predetermined fracture points on the outer surfaces of the extrudates. All extrudates had the form of cylindrical bodies, pieces 1 and $1.5 \mathrm{~cm}$ long and of $0.5 \mathrm{~cm}$ width were selected, and placed on a sheet of paper. A razor blade was lightly pushed onto the surface of the extrudate and the cylindrical body moved and rolled under the razor blade. This gave the extrudate surfaces a pre-determined breaking point, a closedcircular scratch, $\sim 0.5 \mathrm{~mm}$ deep. Fracture was then introduced by bending the extrudates with the use of two tweezers. The freshly fractured extrudates were mounted on an optical glass slide by use of a 2 component epoxy resin, which hardened within $\sim 5 \mathrm{~min}$. Before the hardening reaction had been completed the extrudate orientation was corrected to get the fracture surface as horizontal as possible. This step is mandatory to enable non-destructive imaging and automated sample changing within Atomic Force Microscope operations.

Stability studies were carried out on freshly fractured extrudates. The samples were stored at accelerated stability conditions $\left(\mathrm{T}=40^{\circ} \mathrm{C}, \mathrm{RH}=75 \%\right)$ for $2 \mathrm{~h}$. Before stress storage the mounted extrudates were brought to a temperature of $\mathrm{T}=40^{\circ} \mathrm{C}$ to minimize the risk of unspecific moisture condensation during incubation. The surfaces were stressed in a pre-equilibrated desiccator having a static room humidity $(\mathrm{RH})$ of $75 \%$ (saturated solution of sodium chloride) and a temperature of $\mathrm{T}=40^{\circ} \mathrm{C}$. The stress storage time is the effective storage time at $\mathrm{RH}=75 \%, \mathrm{~T}=40^{\circ} \mathrm{C}$. Finally the samples were quickly removed from the humid, high temperature environment and "dried" in a vacuum chamber.

\section{MIMix Workflow}

The objective was the inspection and comparison of fracture-exposed extrudate interfaces with those described previously for the corresponding QCMM, using the same MIMix workflow as summarized above (26).

\section{Raman Imaging}

Raman mapping experiments were performed on a LabRAM ARAMIS (HoribaJobinYvon) Raman microscope equipped with a Peltier cooled CCD detector using laser excitation at $785 \mathrm{~nm}$ and a $600 \mathrm{l} / \mathrm{mm}$ grating centered at $1,100 \mathrm{~cm}^{-1}$ (wave number range $450-1,730 \mathrm{~cm}^{-1}$ ). For each sample at least $441(21 \times 21)$ spectra were recorded in an area of $80 \times 80 \mu \mathrm{m}^{2}$ using a $50 x$ objective (Olympus; NA 0.75). Settings were chosen to guarantee a spatial resolution of at least $5 \mu \mathrm{m}$ (size of measurement spot for a single
Raman spectrum). All Raman spectra of one mapping experiment were evaluated by integration of bands typical for API or excipient in the mixture. Homogeneity was judged by the ratio variation of API and excipient. The full data set is shown in Supplementary Material.

\section{Atomic Force Microscopy}

Freshly fractured extrudates on microscopic glass slides were mounted on the micrometer positioning stage of a Dimension Icon AFM (Bruker, Santa Barbara). Between 10 and 25 regions per sample were programmed to be automatically characterized using the software routine "programmed move" in Tapping Mode (Bruker Nansocope V8 r1.5). Height, phase, and amplitude images were collected simultaneously, using etched silicon cantilevers with a nominal spring constant of $\mathrm{k}=20-80 \mathrm{~N} / \mathrm{m}$ (Bruker AFM Probes, RTESPA). The typical free vibration amplitude was in the range of $\mathrm{A}=25 \mathrm{~nm}$, the images were recorded with set-point amplitudes corresponding to $60-70 \%$ of the free amplitude. Image areas of $3 \times 3 \mu \mathrm{m}^{2}$ were recorded at a resolution of $1,024 \times 1,024$ pixels. All data were batch-processed using Scanning Probe Image Processor (SPIP 5.1.1). Height data were plane-corrected by applying a $3^{\text {rd }}$ order polynomial fit. Molecular fracture roughness data as displayed in the Figs. 3 and 6 show the root mean square roughness and standard deviation calculated from at least 20 images on each sample. The roughness parameters reflect the variation with respect to the topography mean height. The phase images shown are non-processed raw data.

\section{RESULTS}

Figure 1 shows the chemical structure of the NK1 receptor antagonist, and of the CETP inhibitor. Both APIs had been
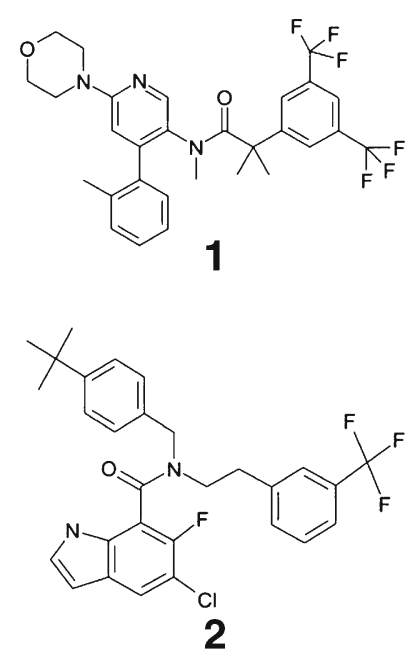

Fig. I Chemical structure of selected APIs. NKI receptor antagonist (I), and CETP inhibitor (2). 
profiled for their intrinsic melt-miscibility within a set of five different excipients (PVP VA64, PVP K30, HPMCAS MF, PVP PF17, Eudragit L100), and at a fixed drug load of $50 \%$. The melt-miscibility had been determined on QCMMs. These were prepared from glassy films, which were melthomogenized at $\mathrm{T}=180^{\circ} \mathrm{C}$ before quench-cooling (26).

These ten combinations were processed here with a small scale Hot-Melt Extruder (HME) as described above. The ten combinations were processed with the same extruder, applying comparable feed and screw speeds, and at a comparable temperature operation window ranging from $\mathrm{T}=120^{\circ} \mathrm{C}$ to $140^{\circ} \mathrm{C}$.

\section{MIMix Homogeneity}

The micrometer homogeneity of the extrudates, which is examined in the first working step of MIMix, is shown in comparison with that of the corresponding QCMMs in Table I. Three of the ten extrudates prepared have an irregular spatial API distribution on the micrometer scale, as examined by Raman Imaging (Supplementary Material). Nanometer homogeneity, which is accessed in the second step of MIMix, is observed in only two of the seven remaining combinations: the NK1(Ant) : PVP PF17, and the CETP(Inh) : HPMCAS MF extrudate. The nanometer homogeneity of all extrudates and of the published QCMMs is shown and compared with the roughness charts in Fig. 2, and the corresponding recorded AFM phase maps in Figs. 3 and 4.

The surface roughness variations quantitatively match those of the QCMMs for the NK1(Ant) : PVP PF17 extrudate (Fig. 2a), and for the CETP(Inh) : HPMCAS MF extrudate, as well as the CETP(Inh) : PVP VA64 extrudate (Fig. 2b). The four other extrudates are different; they are only micrometerhomogenous. In these cases the surfaces are, if compared with the QCMMs, rougher, and/or the roughness measured on different sample regions is divergent. The absolute roughness and/or the regional deviations (variance) are accordingly larger, and do not match that of the corresponding and homogenously constituted QCMM.

However, the AFM phase maps which were recorded on the extrudates additionally indicate that only the NK1(Ant) : PVP PF17 and the CETP(Inh) : HPMCAS MF extrudates fully compare with their corresponding QCMM (Figs. 3d, i and $4 \mathrm{c}, \mathrm{h})$. Only those two extrudates strictly fulfilled all of the introduced MIMix homogeneity criteria.

The GETP(Inh) : PVP VA64 extrudate (Fig. 4a, f) is close to homogeneous, with only a sole nanometer-sized droplet found within the 25 independently inspected surface regions. This is depicted by a Phase-Homogeneity-Ratio (PHR) of $1 / 25$ as directly shown in the phase map (Fig. 4f). The PHR relates the amount of phase maps showing a distinct feature (as shown in the image) with the amount of overall recorded maps (all the other phase maps recorded were featureless, and are not shown here). The PHR indicates how representative the map shown is. All other extrudates are more critically interspersed with nanometric features with many phase maps showing features not typical for homogenous mixtures. Apparently most of the prepared extrudates have not reached the homogeneity that is possible or had been previously achieved with the QCMM preparation.

\section{Process Optimization}

In order to identify whether the homogeneity that is achieved in the QCMMs can also be achieved by means of hot melt extrusion, the $\mathrm{NK} 1$ (Ant) combination with PVP VA64 was extruded at a higher mixing temperature of $\mathrm{T}=160^{\circ} \mathrm{C}$. The surface roughness of this higher temperature extrudate was measured and compared with its heterogeneous analogue extruded at $\mathrm{T}=140^{\circ} \mathrm{C}$, and with the corresponding QCMM results in the Fig. 5.

The higher process temperature results in a similar fracture roughness and variance, a surface status similar to that found using the QCMM (Figs. 2a and 3a). The sample roughness data match quantitatively, with the fractureexposed bulk interfaces of the high temperature extrudate clearly smoother and more uniform than when extruded at $\mathrm{T}=140^{\circ} \mathrm{C}$.

\section{MIMix Stress Test}

The third and last step of the MIMix workflow was to analyze all the homogenously constituted extrudates in respect of their potential to phase separate in a humidity stress environment. We selected here three extrudates, all meeting

Table I Spatial API/Excipient Distribution of Assay and HME Dispersion Systems as Examined with Confocal Raman Imaging

\begin{tabular}{|c|c|c|c|c|c|}
\hline & PVP VA 64 & PVP K 30 & HPMCAS MF & PVP PF 17 & Eudragit L 100 \\
\hline NKI(Ant) assay & homo. & homo. & homo. & homo. & hetero. \\
\hline NKI (Ant) HME & homo. & homo. & hetero. & homo. & hetero. \\
\hline CETP(Inh) assay & homo. & homo. & homo. & homo. & homo. \\
\hline CETP(Inh) HME & homo. & homo. & homo. & homo. & hetero. \\
\hline
\end{tabular}

homo: : uniform API/excipient distribution on the micrometer scale

hetero. non-uniform API/excipient distribution on micrometer scale 


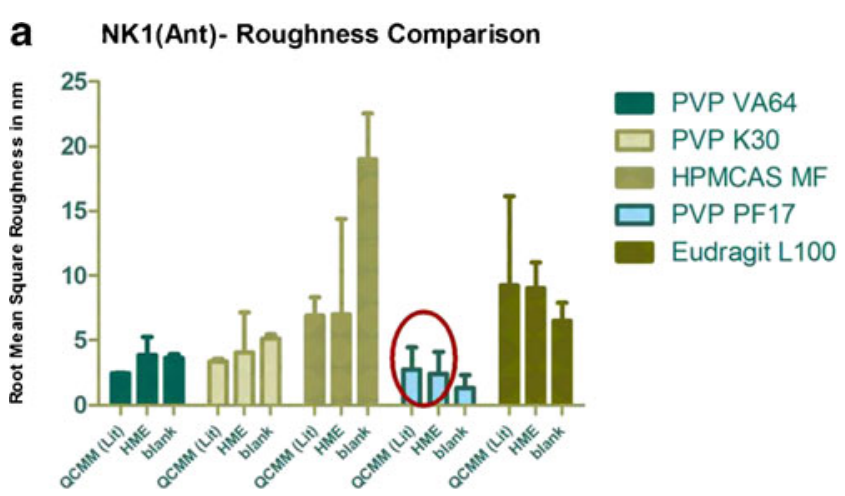

\section{b CETP(Inh) Roughness Comparison}

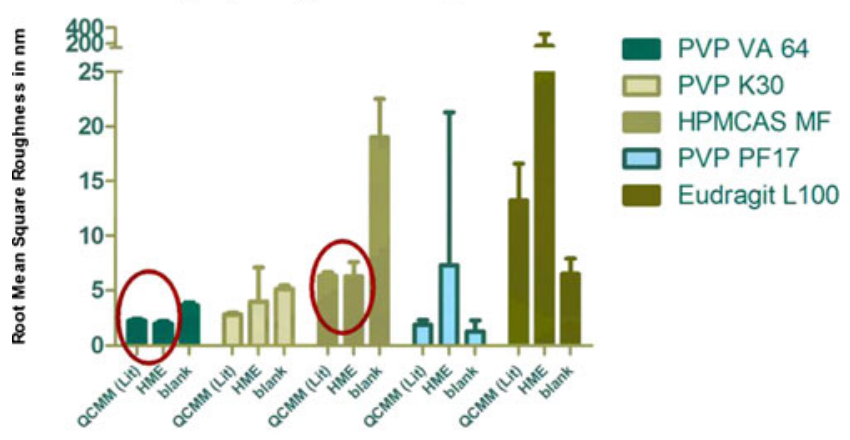

Fig. 2 The Root Mean Square roughness of freshly fractured API-excipient extrudates is compared with that of QCMMs and excipient blanks published previously (25). (a) Root Mean Square Roughness and standard deviations of all investigated NKI(Ant) systems and the corresponding excipient blanks, (b) Root Mean Square Roughness and standard deviations of all CETP(Inh) systems and corresponding excipient blanks.

or almost meeting the MIMix homogeneity criteria (CETP(Inh) : PVP VA, NK1(Ant): PVP PF17, CETP(Inh):HPMCAS MF). These three extrudates were freshly fractured before being exposed to accelerated stress conditions of $\mathrm{RH}=75 \%$, and $\mathrm{T}=40^{\circ} \mathrm{C}$, for $2 \mathrm{~h}$. 10 different locations on each extrudate were then re-inspected by tappingmode AFM for stress-storage introduced phase separation patterns. In Fig. 6 the phase separation patterns observed are compared with those of the correspondingly stressed QCMMs.

The phase separation patterns evolved on the extrudates and QCMM are comparable for each API : excipient system. The patterns do not depend on the way how the systems have been prepared. The NK1(Ant)-based systems had previously been described to undergo spinodal demixing, with the stressed surface uniformly decorated with the characteristic bimodal patterns (Fig. 6a,b). In contrast, the CETP systems tended to undergo de-mixing by nucleation and growth (Fig. 6c-f). Isolated spots can then be observed, which were shown to grow and crystallize with time previously (26). The domain size and separation length of the evolved bimodal patterns on the NK1(Ant) extrudate (Fig. 6b) are comparable with those of the NK1(Ant) QCMMs (Fig. 6a) (26). The size and amount of detected nanoparticles, in relation to the total of the imaged area, is on the CETP-based extrudates comparable with that of the corresponding QCMMs (Fig. 6c-d and e-f). 5 out of 25 imaged regions are spiked with nanoparticles in case of the CETP(2):PVP VA64 QCMM (6e), and 3 out of 15 imaged regions are decorated with particles on the corresponding extrudate (6f). The situation on both CETP(Inh) : HPMCAS MF systems is comparable as well (Fig. 6c-d).

To estimate the fundamental impact of the initial homogeneity status on molecular de-mixing processes within similarly composed systems, the following experiment was performed. The three differently prepared and differently constituted NK1(Ant) : PVP VA64 systems as shown in the Fig. 5, were
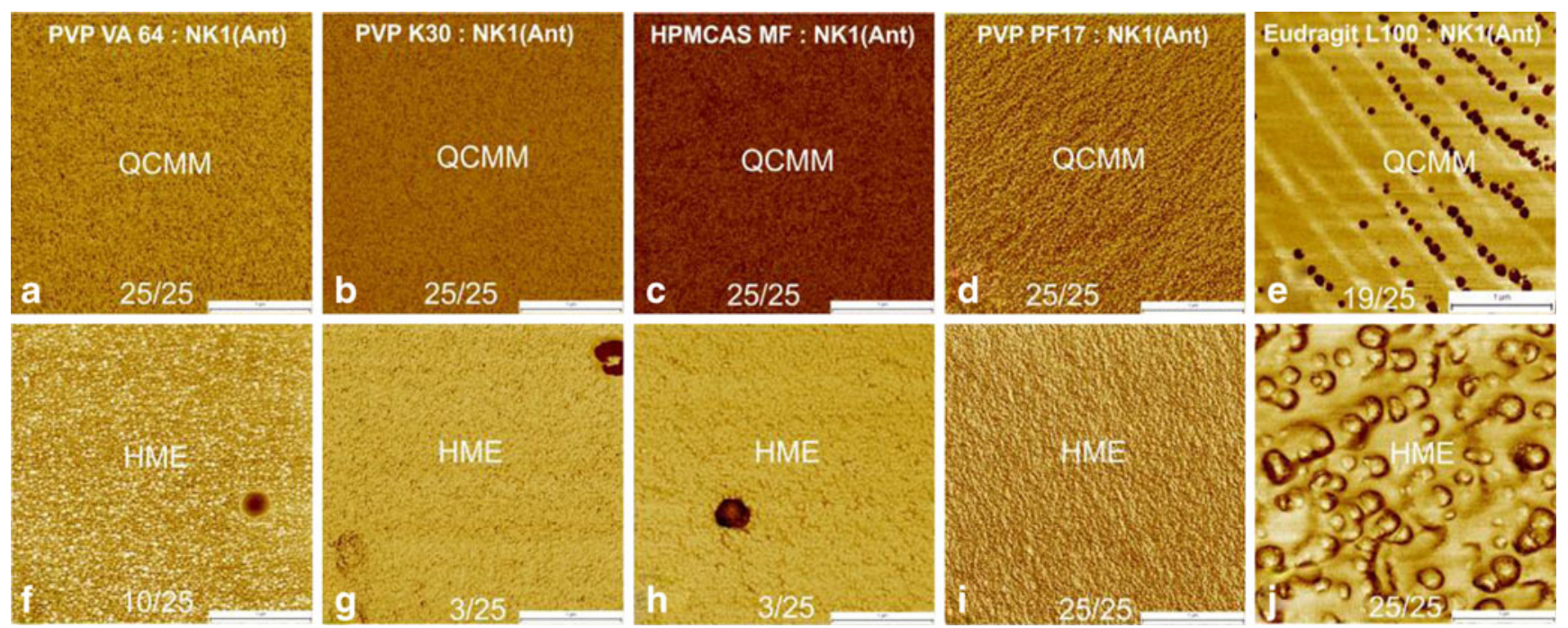

Fig. 3 Nanometer homogeneity of freshly fractured NKI(Ant) QCMMs (top row) is compared with corresponding extrudates phase maps as they were recorded with tapping-mode AFM: top row (a-e) was recorded on the QCMMs published previously, bottom row is (f-j) recorded on the corresponding extrudates. The numbers visible in the image rows (a-e, and $\mathbf{f}-\mathbf{j}$ ) relate the amount of comparably structured places with the amount of investigated area (PHR). 

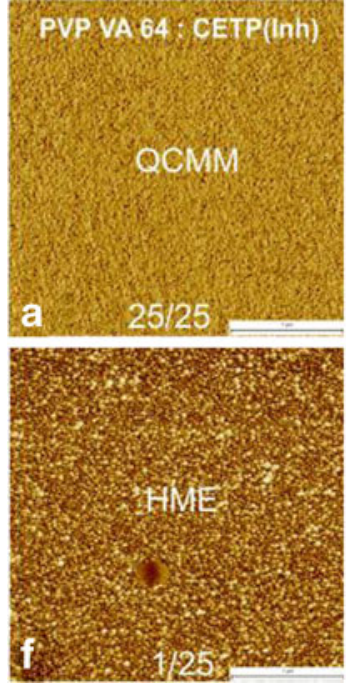
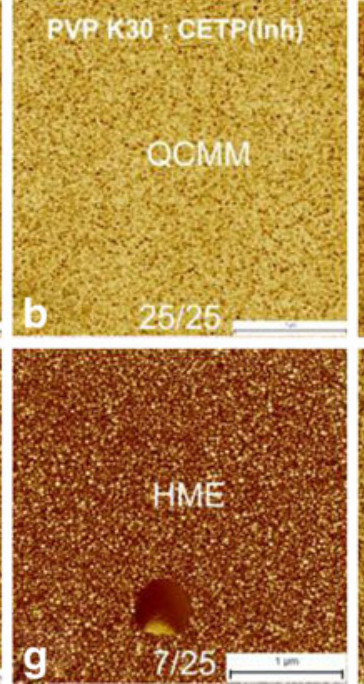
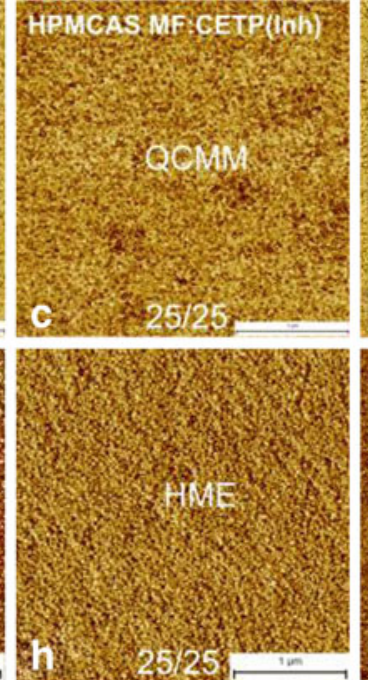
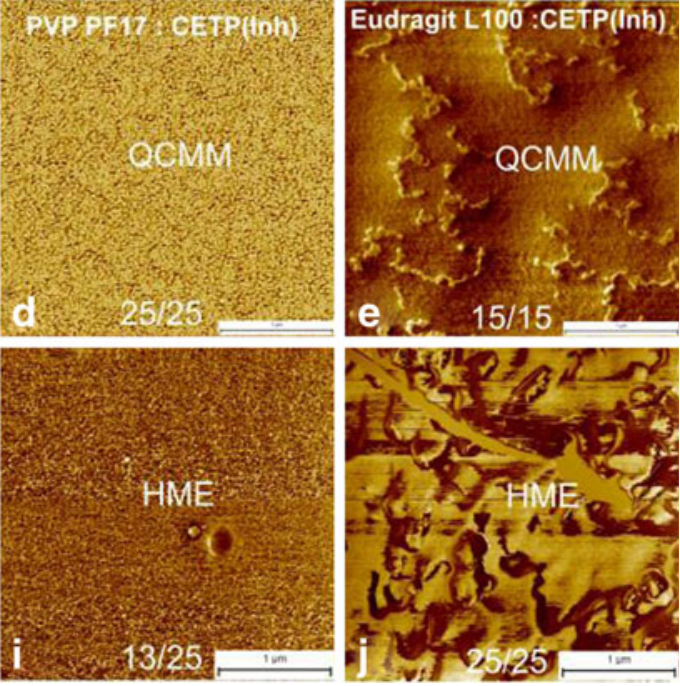

Fig. 4 Nanometer homogeneity of freshly fractured CETP(Inh)-QCMMs (top row) is compared with corresponding extrudate, phase maps as they were recorded with tapping-mode AFM: top row (a-e) was recorded on the QCMMs published previously(f-j), bottom row is recorded on the corresponding extrudates. The numbers visible in the image rows (a-e, and $\mathbf{f}-\mathbf{j})$ relate the amount of comparably structured places with the amount of investigated area (PHR).

stressed for $2 \mathrm{~h}$ at $\mathrm{RH}=75 \%$, and $\mathrm{T}=40^{\circ} \mathrm{C}$. The evolved decomposition patterns are compared side by side in Fig. 7.

The bimodal patterns of the QCMM (Fig. 7a) and of the homogenous extrudate (Fig. 7b) are comparable. The surface of the heterogeneous/low-temperature extrudate is different (Fig. 7c). After stress storage the initial, frequently noticed, droplet-like spots (Fig. 3f), have become decorated with particles of needle like or grainy morphology (Fig. 7c). These particles often provide a detectably harder mechanical contrast within the AFM phase maps (harder sample regions can appear whiter in the maps). Taken together it appears, that the de-mixing process of a API:excipient

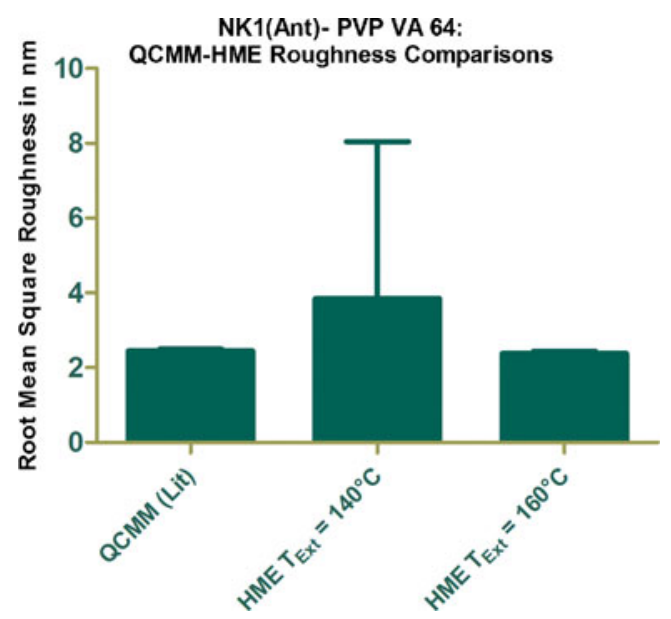

Fig. 5 Homogeneity comparison of similarly composed and differently processed NKI(Ant)-PVP VA64 dispersions on basis of their molecular surface roughness. composition depends on both the possible homogeneity status of the dispersion system and on the process history.

To roughly estimate the macroscopic relevance of our nanometers-scaled and surface-restricted AFM studies, the overall quick aging NKl(Ant) extrudates (independent of whether they were homogenous or not) were subjected to 3 month-scaled stress exposures $\left(\mathrm{RH}=75 \%, \mathrm{~T}=40^{\circ} \mathrm{C}\right)$. Photographs of the stressed extrudates were recorded. In Fig. 8 the published assay ranking results for the NK1 systems (Fig. 8e), are compared with the macroscopically observed whitening of their corresponding extrudate photographs taken after 3 months.

The HPMCAS MF-based NK1(Ant) system showed slower de-mixing than all PVP-based sample systems (Fig. 8e). The HPMCAS extrudate preserved its glassy appearance on the millimeter scale (Fig. 8a). In contrast the PVP K30-based system, which de-mixed rapidly in the published assay, is found from the macroscopic perspective to be clearly de-mixed (Fig. 8d, e).

\section{DISCUSSION}

Amorphous solid dispersion is an umbrella term for different kinds of amorphous mixtures, often classified by their thermodynamic phase behavior. In pharmaceutical research, solid dispersions are also distinguished in regard to their dissolution properties (30). Binary drug-polymer mixtures, when they have been prepared by solvent casting, typically belong in the class of "transparent glassy amorphous solid solutions". To absolutely and objectively compare differently composed, structured or prepared solid dispersions, previously only characterized with purely static imaging data, 
Fig. 6 Comparison of phase separation processes of the homogenously constituted QCMM and extrudate systems after exposure to stress conditions $(\mathrm{RH}=75 \%, \mathrm{~T}=$ $40^{\circ} \mathrm{C}$ ) for $2 \mathrm{~h}$. The initial and homogenous states are shown in Figs. 4 and 5 for comparison. The numbers visible in the images relate the amount of comparably structured places with the amount of investigated area (PHR).
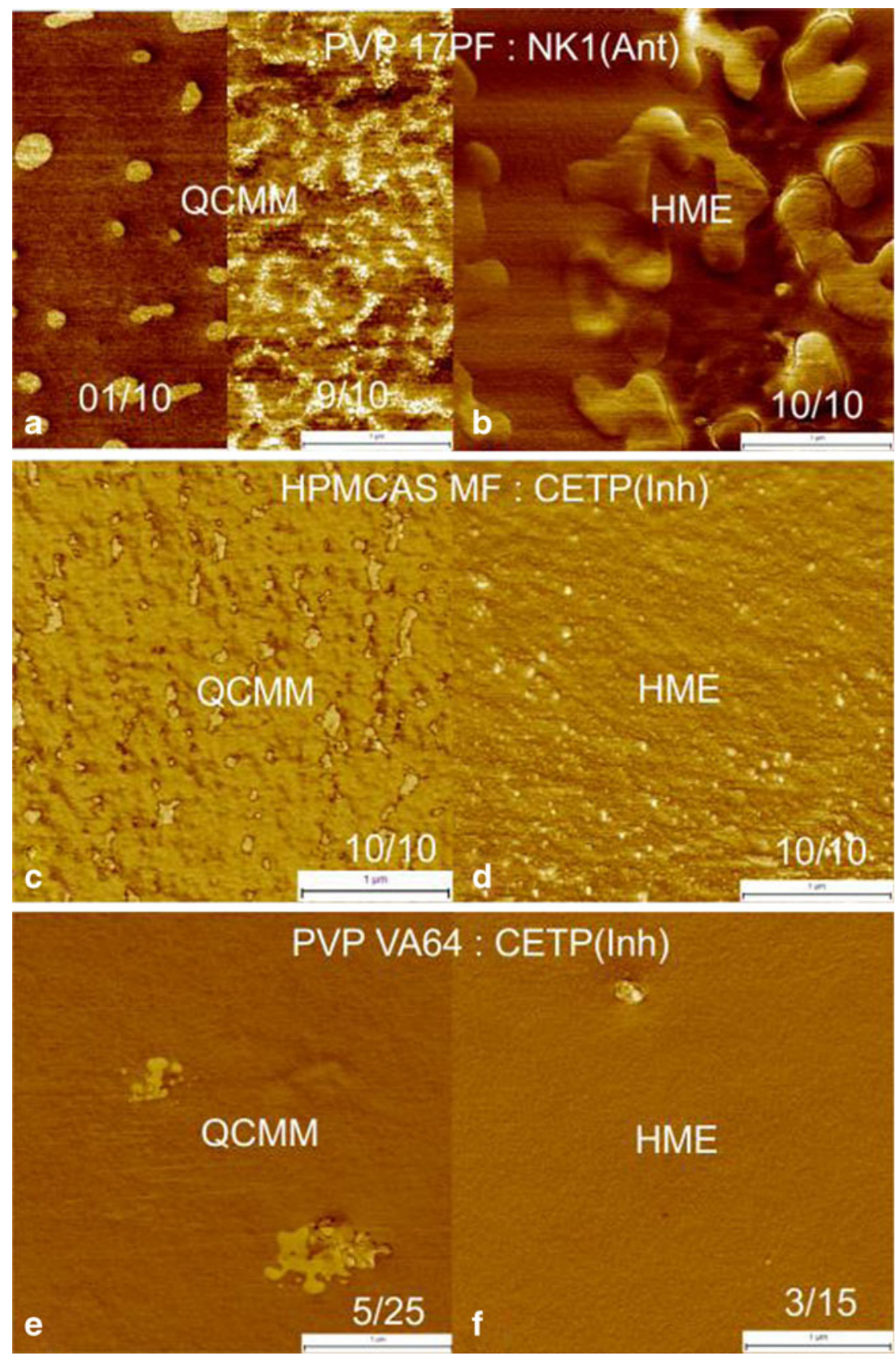

another and phenomenological classification concept was applied.

\section{MIMix Classification System}

Analyzing all the AFM phase data recorded within this study (> 600 phase maps), only three different types of molecular heterogeneity constitutions could be recognized (Fig. 9a): Class A1, Class A2, and Class B.

\section{Class AI Dispersion}

Both components are tightly and continuously intermixed on the nanometer scale. They fulfill the MIMix homogeneity criteria and are characterized by uniform and flat phase maps. Their surface roughness and variance is typically smaller than that of the applied excipient alone. We will introduce the term "continuously-homogenously mixed systems" for these types of solid dispersion.

\section{Class A2 Dispersion}

The excipient matrix is mainly continuously structured; however, critical spots are occasionally recognized. The appearance of local spots on a subset of recorded images is indicated by a PHR not equal to one. A2-class systems have a surface roughness and variance exceeding that of the Al-class dispersions. They are here termed "discontinuously-mixed solid dispersion systems". 

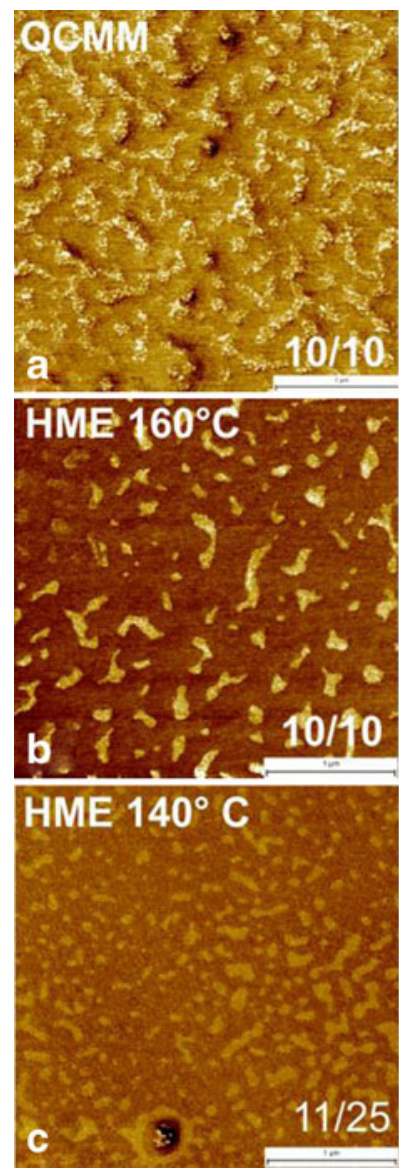

Fig. 7 Comparison of phase separation processes of differently prepared NKI(Ant):PVP VA64 systems after exposure to stress conditions $(\mathrm{RH}=$ $75 \%, \mathrm{~T}=40^{\circ} \mathrm{C}$ ) for $2 \mathrm{~h}$ (samples of Fig. 6): (a) pattern as observed on QCMMs, (b) patterns as observed on homogenously constituted HME samples prepared at $T=160^{\circ} \mathrm{C}$, (c) pattern as observed on a heterogeneously constituted $\mathrm{HME}$ sample prepared at $\mathrm{T}=140^{\circ} \mathrm{C}$. The numbers visible in the images relate the amount of comparably structured places with the amount of investigated area (PHR).

\section{Class B Dispersion}

This third group contains all dispersion systems of a continuousheterogeneous phase constitution: they can be termed "de-mixed solid dispersion systems". The surface roughness and variance can exceed that of the polymer systems, and all phase maps are of continuously heterogeneous contrast $(\mathrm{PHR}=1)$.

The MIMix homogeneity classes (A1, A2, and B) now enable a better assessment of the efficiencies of the two different preparation processes. If the QCMMs are compared pairwise with the extrudates, only three of the nine theoretically possible class combinations are observed: A1-A1, A1-A2, and B-B (QCMM - HME), as illustrated in Fig. 9b-c. Combinations such as B-A1, or A2-A1 do not appear. In other words, it was so far not possible to record data on the extrudates where the MIMix homogeneity is better than of the QCMMs. They have a higher probability to reflect the intrinsically possible
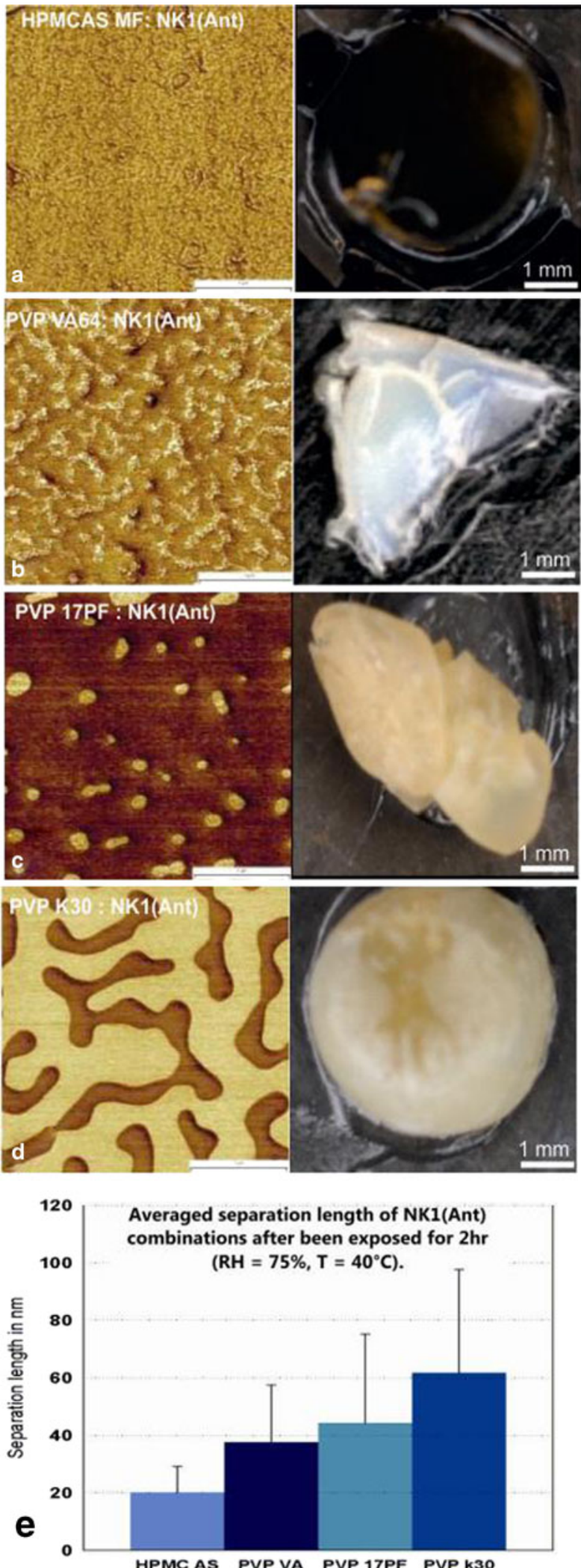

Fig. 8 Phase separation patterns as observed on NKI (Ant) QCMMs after $2 \mathrm{~h}$ scaled stress exposure to $\mathrm{RH}=75 \%, T=40^{\circ} \mathrm{C}$ (left column) are opposed with photographs of corresponding extrudates stressed at similar conditions for 3 months (a-d). (e) Phase separation velocities and stability ranking as communicated previously. 
Fig. 9 MIMix classification system. (a) Schematic phase maps as they are typically recorded on solid dispersion systems with MIMix are filed in three classes: continuoushomogenous (Al), discontinueshomogenous (A2), continuousheterogeneous (B). (b) list of QCMM: extrudate class combinations so far observed with MIMix. (c) Scheme to illustrate the class combinations theoretically possible.

(Combinations that have been observed are outlined yellow.)

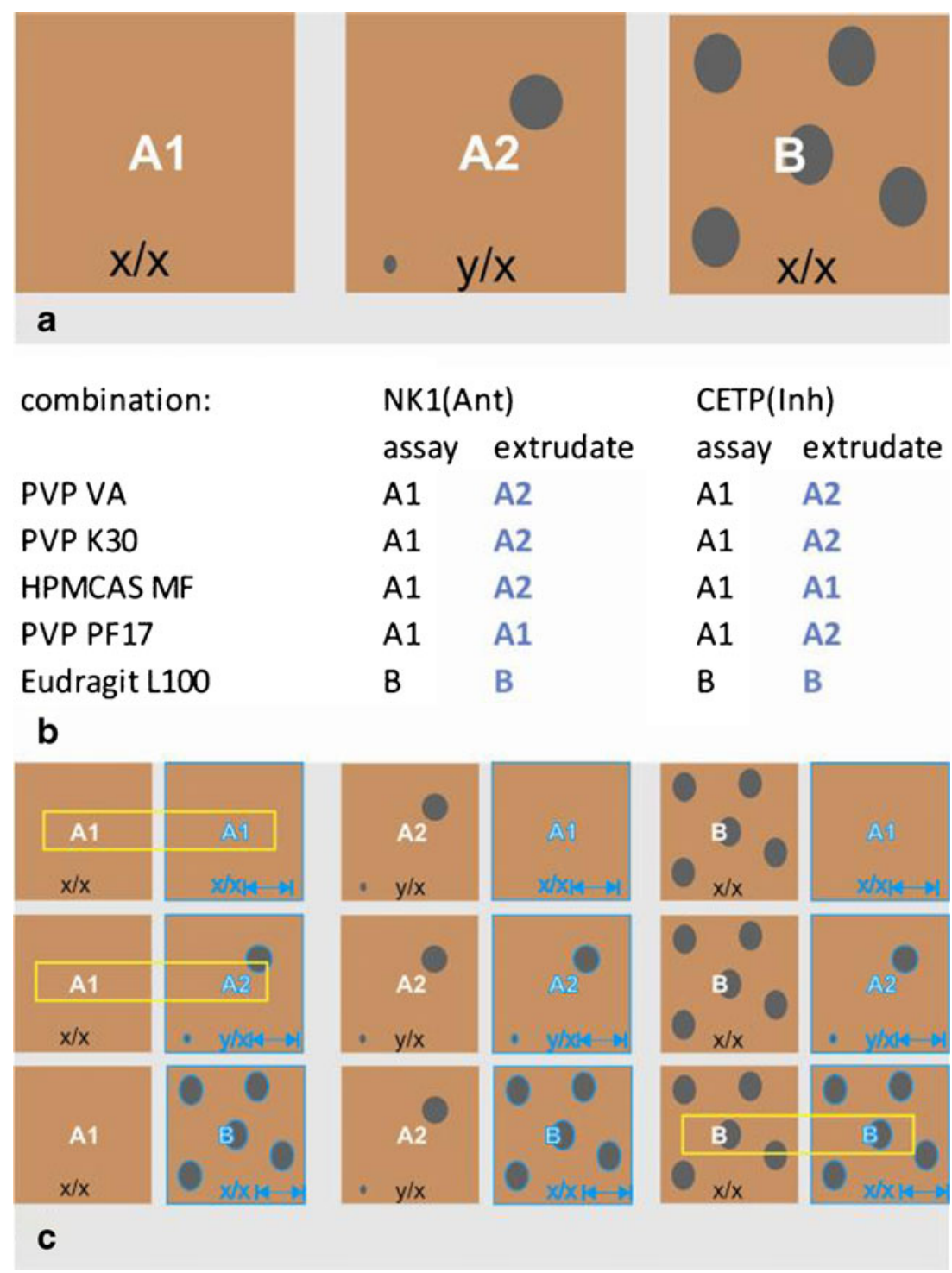

miscibility/immiscibility of a given API within the excipient systems considered. This fundamental finding supports our previous hypothesis that melt-homogenized solvent cast films are likely to reflect the intrinsic miscibility of the considered API:excipient in a melt state (26). This hypothesis has recently been verified on Naproxen/PVP K25 solid dispersions (31).

The QCMMs are thus not only a practical preparation system for screening purposes (26), but also their molecular homogeneity status reflects most likely a miscibility optimum. As the MIMix homogeneity data of QCMMs and extrudates can be compared side by side, they are a novel basis to benchmark the homogeneity basically accessible with an optimized HME process. Taken together the knowledge is very helpful to judge and to compare objectively the efficiencies of differently conducted HME processes.

An Al-Al situation indicates that the HME process has reached the homogeneity that is close to the API : excipient miscibility in a melt state. Melt miscibility was only realized here in the NK1(Ant) - PVP PF17, and the CETP(Inh) HPMCAS MF extrudates. Most other extrudates belong to class A2. The homogeneity achieved in the extrusion process is inferior to that possible in the melt state (Fig. 9b). Accordingly, A1-class extrudates are the desired ones. Extrudates of class A2 must not be excluded from further formulation development, but the HME process needs to be refined. Extrudates assigned to class B were so far not suitable.

The PHR is a parameter which may be used to estimate the details and differences of different A2 classes in a semiquantitative manner. The NK1(Ant) : PVP VA64 extrudate is, for example, when compared with the NK1(Ant) : PVP K30 system, more heterogenous. 13 out of 25 recorded phase maps show separated spots, with only 3 out of the 25 recorded maps in the PVP-K30-based system. The PHR enables an extension of the classification system introduced. The NK1(Ant) : PVP64 extrudate can be said to exhibit an A2-(13/25) state, whereas the NK1(Ant): PVP K30 
extrudate shows an A2-(3/25) state. This MIMix classification system provides a simple basis to better oversee, compare, track, and communicate such comparatively huge sets of phase maps in an easy and direct way.

\section{Process Refinement, Roughness Analysis}

Many different aspects need to be considered to identify the most appropiate excipient in order to prepare a well performing amorphous solid dispersion. A large scale HME processes is, however, defined at a fixed drug load, and within an already identified excipient system. The surface roughness, which is most practically to determine on QCMMs, is a quantitative parameter to benchmark and to compare absolutely and quantitatively the efficiency of differently conducted extrusion processes (Fig. 5). This is demonstrated here on the NK1(Ant) - PVP VA64 - based systems. The surface roughness of the high temperature extrudate quantitatively matches that measured on the QCMM with impressive precision (Fig. 5), the same extrudate is MIMix heterogeneous (A2 class) when extruded at $\mathrm{T}=140^{\circ} \mathrm{C}$ (Fig. 5).

The MIMix roughness concept of excipients and of Alclass mixtures can be understood intuitively with a simplistic physical model, which is based upon the fundamental concept of the free volume theory of polymers: the polymer as excipient has a much larger size than a usual API. Fracture-exposed bulk interfaces consisting of large building blocks are, for purely geometric reasons, rougher (Fig. 10b) than those constituted exclusively from small molecules. Al-class mixtures, when the polymer matrix is homogenously inter-diffused with small molecules, typically expose smoother bulk interfaces than pure polymer excipients (Fig. 10a). The drug molecules can act as small "gap-fillers" (32,33). The A2-class and B-class mixtures are interspersed with nanoparticles of several API molecules. If such particles reach a size range comparable to, or bigger, than that of the polymer molecules, the fracture surface becomes rougher. Different regions of the sample will show a bigger variability (Fig. 10c).

This purely geometric packing consideration is a coarse approximation. It can model most of our results in a

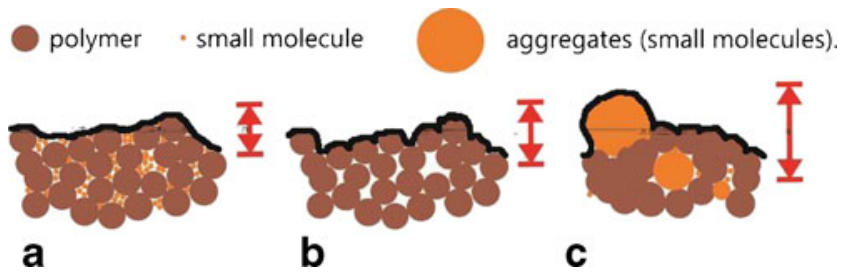

Fig. I0 Simplified model to illustrate the concept of surface roughness and the drug smoothening effect on homogenous mixtures. (a) Models a homogenous situation, the smaller molecules are dispersed between the bigger polymer molecules. (b) Models the situation of polymer blank. (c) Models a heterogeneous situation and shows domains of small molecule aggregates bigger than the polymer. phenomenological manner. A comprehensive model would need to take additional factors like the excipient and API interactions, the conformational freedom of differently sized or structured polymers, the rigidity or persistence of polymeric backbones, the fracture mechanics and possible surface relaxation processes, which can be expected to occur during or after fracturing, into account. They are here not discussed further (34).

However, from the pragmatic point of view the magnitude of the surface roughness variation expresses the specific physical properties of the excipient/polymer systems considered: in all API:excipient mixtures investigated here, it does not noticeably depend on the nature of the API. The roughness data of all A1-class NK1(Ant) mixtures quantitatively compare with those of the corresponding A1-class CETP(Inh)-based mixtures (Fig. 2a-b). The roughness analysis can deliver a novel parameter allowing to better compare the molecular homogeneity of differently prepared systems in a reproducible manner. However, the parameter is only helpful as long as dispersion systems of one and the same polymer system are considered, where the roughness can be compared in absolute terms.

\section{Stress Tests and Molecular Phase Separation Rates}

Accelerated stability studies are generally used to identify appropriate excipients for formulation development. The AFM-based assay here tested all the extruded combinations in this respect. To verify whether the published data of the QCMMs indeed reflect a reproducible and intrinsic potential of the API: excipient system considered, the same stability study was carried out on extrudates. Two hour scaled stress exposure was already sufficient to recognize on the nanometer scale the different potentials of the extrudates to physically demix when humid (Fig. 6). However, only the Al-class extrudates compare well in detail with their corresponding QGMMs. Firstly, in a qualitative way, as identical phase separation mechanisms are observed: spinodal phase separation patterns were seen on all NK1 (Ant)-QCMMs (Fig. 6 a, b), whereas, in contrast, the CETP(Inh)-QCMMs de-mixed by nucleation and growth (Fig. 6c-f). Secondly, they compare in a quantitative way, as the amount of separated particles (nucleation and growth) or the separation distances (spinodal phase separation) are comparable. A2 and B-class extrudates, however, are more poorly defined and are found difficult to reproduce experimentally (Fig. 7).

The possible impact of the MIMix homogeneity on the molecular phase separation mechanism is shown explicitly for the different NK1(Ant):PVP VA64 systems. The homogeneity status of these three differently prepared systems is depicted in Fig. 5. The decomposition patterns evolving on the Al-class extrudate (processed at the higher temperature of $\mathrm{T}=160^{\circ} \mathrm{C}$ ) match with those of the QCMM. The A2-class extrudate 
(extruded at $\mathrm{T}=140^{\circ} \mathrm{C}$ ) is different. The stressed A2-class extrudate frequently shows crystallized droplets and features. (Fig. 8c), and the bimodal patterns are likewise variable.

If some API is initially condensed within droplets before the exposure to stress storage, the dispersion matrix lacks this amount of API. The potential of a mixture to phase separate, however, directly depends on the API concentration in the excipient matrix. It is clear from a fundamental point of view that an already de-mixed system (A2- and B-class) inevitably demixes, ages and performs differently than its A1-class analogues.

The MIMix stability data recorded in Al state QCMMs reflect a reproducible potential of the API: excipient system considered (Fig. 7). However, solid dispersions which do not have an identical homogeneity status in the beginning, age differently. The MIMix homogeneity data, the roughness and the classification concept introduced are fundamental for such studies. They enable observation, identification and comparison of such small structural differences in a consistent and direct manner.

As most of the extrudates represented A2 homogeneity states, it was not straightforward to carefully validate and compare their physical stability using bulk analytical techniques. Overall the NK1 systems age more rapidly than the CETP-based systems. The underlying phase separation mechanism, which is a spinodal phase separation process in $\mathrm{NK} 1$ (Ant)-based systems seems to be the reason. In a spinodal process the whole material surface has to de-mix uniformly and in concerted fashion. A larger amount of API can be expected to segregate in a short amount of time (35), and nucleation and growth acts only locally (36). To roughly estimate the basic validity of our MIMix prediction concept, all the NK1 extrudates (whether they are molecularly homogenous or not) were exposed for 3 months to acceleratedstress conditions. Photographs were then recorded (Fig. 8). This purely phenomenological approach is a direct and simple way to identify materials which are obviously demixed on macroscopic length scales. Phase separating glasses undergo whitening and, over time, become turbid.

The NK1(Ant) : HPMCAS MF was previously predicted to be the most stable NK1(Ant) combination (Fig. 8a). The corresponding photographed extrudate maintained the overall glassy appearance macroscopically. All the PVPbased extrudates underwent whitening, apparently gradually. They are more turbid. The AFM data - photograph comparison in Fig. 8 reminds us, that even the 2dimensional restricted phase separation processes depend on the specific and intrinsic phase separation potentials of the API: excipient system considered (37). These potentials can comparatively be studied with the AFM in few hours on representative model surfaces, on QCMMs. The MIMix stability data are thus an analytical basis to rapidly identify API: excipient systems having a low potential to phase separate on the long term.

\section{CONCLUSIONS}

The MIMix concept allows the verification, comparison and classification of the molecular homogeneity of differently composed, constituted or processed amorphous solid dispersions in a direct and rational manner. Two different types of observation, which can easily be accessed by tapping-mode AFM, are found to be robust enough. Firstly, AFM phase maps, which directly visualize the phase homogeneity constitution of the mixture, and, secondly, AFM height maps, which allow to determine the specific molecular surface roughness of the API: excipient system considered.

The MIMix classification system enables the side by side comparison of various solid dispersion systems and the identification of sources of molecular heterogeneity. Heterogeneity, which can be caused by poorly miscible API: excipient combinations, can be distinguished from heterogeneity introduced by a poorly optimized HME process. MIMix roughness analysis is a convenient tool to compare quantitatively the molecular homogeneity within one and the same excipient system when samples are prepared by different means. The roughness analysis is helpful to guide the selection and refinement of a given extrusion process at the technical level.

MIMix stability data, which can be gathered in miniaturized QCMMs within only hours, compare well with the stability of the corresponding extrudate system observed not just after hours but also after months. The general applicability of AFM and the AFM assay to rapidly identify API:excipient systems which are physically stable on the long term should be taken into consideration wherever possible.

\section{ACKNOWLEDGEMENTS AND DISCLOSURES}

We here like to thank Reto Maurer and Jochem Alsenz for inspiring discussions, and David W. Banner for proofreading the manuscript.

Open Access This article is distributed under the terms of the Creative Commons Attribution License which permits any use, distribution, and reproduction in any medium, provided the original author(s) and the source are credited.

\section{REFERENCES}

1. Williams III RO, Watts AB, Miller DA (Eds): Formulating poorly soluble drugs. New York USA: Springer; 2012.

2. Forster A, Hempenstall J, Tucker I, Rades T. Selection of excipients for melt extrusion with two poorly water-soluble drugs by solubility parameter calculation and thermal analysis. Int J Pharm. 2001;226:147-61.

3. Forster A, Hempenstall J, Tucker I, Rades T. The potential of small-scale fusion experiments and the Gordon-Taylor equation to 
predict the suitability of drug/polymer blends for melt extrusion. Drug Dev Ind Pharm. 2001;27:549-60.

4. Van Eredenbrugh B, Taylor LS. Small scale screening to determine the ability of different polymers to inhibit drug crystallization upon rapid solvent evaporation. Mol Pharm. 2010;7:1328-37.

5. Weuts I, Van Dycke F, Voorspoels J, De Cort S, Stokbroekx S, Leemans R, et al. Physicochemical properties of the amorphous drug, cast films, and spray dried powders to predict formulation probability of success for solid dispersions: etravirine. J Pharm Sci. 201 1;100:260-74.

6. Barillaro V, Pescarmona PP, Van Speybroeck M, Do Thi T, Van HumbeeckJ, Vermant J, et al. High-throughput study of phenytoin solid dispersions: formulation using an automated solvent casting method, dissolution testing, and scaling-up. J Comb Chem. 2008;10:637-43.

7. Ivanisevic I, Bates S, Chen P. Novel methods for the assessment of miscibility of amorphous drug-polymer dispersions. J Pharm Sci. 2009;98:3373-86.

8. Newman A, Engers D, Bates S, Ivanisevic I, Kelly RC, Zografi G. Characterization of amorphous api:polymer mixtures using x-ray powder diffraction. J Pharm Sci. 2008;97:4840-56.

9. Bairdand JA, Taylor LS. Evaluation of amorphous solid dispersion properties using thermal analysis techniques. Adv Drug Deliver Rev. 2012;64:396-421.

10. Qian F, Huang J, Zhu Q Haddadin R, Gawel J, Garmise R, et al. Is a distinctive single T-g a reliable indicator for the homogeneity of amorphous solid dispersion? Int J Pharm. 2010;395:232-5.

11. Sawyer LC, Grubb DT, Meyers GF. Polymer Microscopy. 3rd ed. New York USA: Springer; 2008.

12. Palermo RN, Anderson CA, Drennen JK. Review: use of thermal, diffraction, and vibrational analytical methods to determine mechanisms of solid dispersion stability. J Pharm Innov. 2012;7:2-12.

13. Nollenberger K, Gryczke A, Meier C, Dressman J, Schmidt MU, Bruhne S. Pair distribution function X-Ray analysis explains dissolution characteristics of felodipine melt extrusion products. J Pharm Sci. 2009;98:1476-86.

14. Karavas E, Georgarakis M, Docoslis A, Bikiaris D. Combining SEM, TEM, and micro-Raman techniques to differentiate between the amorphous molecular level dispersions and nanodispersions of a poorly water-soluble drug within a polymer matrix. Int J Pharm. 2007;340:76-83.

15. Magonov SN, Reneker DH. Characterization of polymer surfaces with atomic force microscopy. Annu Rev Mater Sci. 1997;27:175-222.

16. Garcia R, Herruzo ET. The emergence of multifrequency force microscopy. Nat Nanotechnol. 2012;7:217-26.

17. Lamm MS, Simpson A, McVevin M, Frankenfeld C, Nay R, Variankaval N. Probing the Effect of Drug Loading and Humidity on the Mechanical Properties of Solid Dispersions with Nanoindentation: Antiplasticization of a Polymer by a Drug Molecule. Mol Pharmaceut. 2012;9:(11):3396-402.

18. Hu SQ Mininni L, Hu Y, Erina N, Kindt J, Su CM. High-Speed Atomic Force Microscopy and Peak Force Tapping Control. Proc Spie. 2012;11:8324-30.

19. Zhang JX, Bunker M, Chen XY, Parker AP, Patel N, Roberts CJ. Nanoscale thermal analysis of pharmaceutical solid dispersions. Int J Pharm. 2009;380:170-3.

20. Van Eerdenbrugh B, Lo M, Kjoller K, Marcott C, Taylor LS. Nanoscale mid-infrared imaging of phase separation in a drugpolymer blend. J Pharm Sci. 2012;101:2066-73.

21. Mahlin D, Berggren J, Alderborn G, Engstrom S. Moistureinduced surface crystallization of spray-dried amorphous lactose particles studied by atomic force microscopy. J Pharm Sci. 2004;93:29-37.

22. Marsac PJ, Rumondor ACF, Nivens DE, Kestur US, Stanciu L, Taylor LS. Effect of temperature and moisture on the miscibility of amorphous dispersions of felodipine and poly(vinyl pyrrolidone). J Pharm Sci. 2010;99:169-85.

23. Priceand R, Young PM. Visualization of the crystallization of lactose from the amorphous state. J Pharm Sci. 2004;93:155-64.

24. Qi S, Belton P, Nollenberger K, Gryczke A, Craig DQM. Compositional analysis of low quantities of phase separation in hotmelt-extruded solid dispersions: a combined atomic force microscopy, photothermal fourier-transform infrared microspectroscopy, and localised thermal analysis approach. Pharmaceut Res. 2011;28:2311-26.

25. Meeus J, Chen XY, Scurr DJ, Ciarnelli V, Amssoms K, Roberts CJ, et al. Nanoscale surface characterization and miscibility study of a spray-dried injectable polymeric matrix consisting of poly(lactic-co-glycolic acid) and polyvinylpyrrolidone. J Pharm Sci. 2012;101:3473-85.

26. Lauer ME, Grassmann O, Siam M, Tardio J, Jacob L, Page S, et al. Atomic force microscopy-based screening of drug-excipient miscibility and stability of solid dispersions. Pharmaceut Res. 2011;28:572-84.

27. Conte-Mayweg A, Kuehne H, Luebbers T, Maugeais C, Mueller W, Pflieger Philippe, inventor; F. Hoffmann La Roche Ltd. Assignee. Indole, Indazole or Indoline Derivatives. United States patent 7259183. 2007.

28. Hoffmann-Emery F, Hilpert H, Scalone M, Waldmeier P. Efficient synthesis of novel NK1 receptor antagonists: selective 1,4-addition of grignard reagents to 6-chloronicotinic acid derivatives. J Org Chem. 2006;71:2000-8.

29. Van Eerdenbrugh B, Baird JA, Taylor LS. Crystallization tendency of active pharmaceutical ingredients following rapid solvent evaporation-classification and comparison with crystallization tendency from undercooled melts. J Pharm Sci. 2010;99:3826-38.

30. Chiou L, Riegelman S. Pharmaceutical applications of solid dispersion systems. J Pharm Sci. 1971;60:1281-302.

31. Paudeland A, Van den Mooter G. Influence of solvent composition on the miscibility and physical stability of naproxen/PVP K 25 solid dispersions prepared by cosolvent spray-drying. Pharmaceut Res. 2012;29:251-70.

32. Budd PM, McKeown NB, Fritsch D. Free volume and intrinsic microporosity in polymers. J Mater Chem. 2005;15:1977-86.

33. Berggrenand J, Alderborn G. Effect of polymer content and molecular weight on the morphology and heat- and moisture-induced transformations of spray-dried composite particles of amorphous lactose and poly(vinylpyrrolidone). Pharm Res. 2003;20:1039-46.

34. Yang H, Mittal V: Molecular Dynamic Simulation Studies of Binary Blend Miscibility in Functional Polymer Blends, Synthesis, Properties and Performance. 6000 Broken Sound Parkway NW,. Suite 300, Boca Raton, FL 33487: CRG Press, Francis \& Taylor Group: 2012.

35. Mitra MK, Muthukumar M. Theory of spinodal decomposition assisted crystallization in binary mixtures. J Chem Phys. 132: (2010).

36. Sagui C, Grant M. Theory of nucleation and growth during phase separation. Phys Rev E. 1999;59:4175-87.

37. Jones RAL, Norton LJ, Kramer EJ, Bates FS, Wiltzius P. Surface-directed spinodal decomposition. Phys Rev Lett. 1991;66:1326-9. 